\title{
Anthropometric parameters as indicators of metabolic derangements in schizophrenia patients stabilized on olanzapine in an Indian rural population
}

\author{
Jayanta Kumar Rout, Anindya Dasgupta, Om Prakash Singh, Ushasi Banerjee, Brahmarshi Das \\ Department of Biochemistry, Burdwan Medical College, Burdwan, W.B., India
}

\begin{abstract}
Context: For any given body mass, Asian Indians have higher central obesity than Europeans. A periodic measurement of body mass index (BMI) and waist hip ratio (WHR) is practically more feasible than other parameters of metabolic syndrome by repeated blood collection. However, few studies are available on the relative importance of BMI and WHR as markers of dyslipidemia and insulin resistance in schizophrenia patients stabilized on second generation antipsychotics in Indian population. Aim: We conducted the present study on such patients to examine whether BMI or WHR can better predict dyslipidemia and insulin resistance in these patients in a rural area. Settings and Design: The study was a hospital based case control study under rural settings on 38 schizophrenia patients stabilized on olanzapine and 30 matched controls. Materials and Methods: Fasting concentrations of blood glucose, lipid parameters and serum insulin were assessed. Data for Homeostatic model for assessment of insulin resistance (HOMAIR), BMI, and WHR were obtained to assess the insulin resistance, overall body fat distribution and abdominal fat dispensation respectively. Statistical analysis used: ' $t$ ' test was performed to assay any difference in corresponding mean values between cases and controls. Dependence of HOMA-IR on key parameters was assessed by analysis of co-variance (ANCOVA) study. Results: Cases exhibited significantly higher values for HOMA-IR, serum triglyceride and low density lipoprotein cholesterol (LDLc) with a significantly lower high density lipoprotein cholesterol (HDLc) level. ANCOVA study reflected that irrespective of age and sex, HOMA-IR was dependent on serum triglyceride level and WHR ( $F=8.3$ and 5.7 respectively, $P<0.05)$, but not on BMI $(F<0.001, P=0.997)$. Conclusions: Central obesity could be more closely associated with the pathogenesis of prediabetic state in our case group. So, WHR is a better anthropometric parameter than BMI for an early assessment of insulin resistance and dyslipidemia in schizophrenia patients stabilized on olanzapine in our region.
\end{abstract}

Key words: Schizophrenia, insulin resistance, dyslipidemia, waist hip ratio, body mass index

\section{Introduction}

People suffering from schizophrenia show higher rate of mortality in most regions of the world..$^{[1-3]}$ Increased risks for development of metabolic syndrome, lipid abnormalities and consequent cardiovascular deaths have been reported as important contributing factors. ${ }^{[4,5]}$

\begin{tabular}{|l|l|}
\hline \multicolumn{2}{|c|}{ Access this article online } \\
\hline Quick Response Code: & Website: \\
\hline & www.ruralneuropractice.com \\
\cline { 2 - 2 } & \\
\hline
\end{tabular}

Cardiovascular disease remains one of the primary causes of mortality among patients with severe mental illness, with standardized mortality ratios approximately two times greater than the general population. ${ }^{[6]}$ Baseline data from the Clinical Antipsychotic Trials of Intervention Effectiveness ${ }^{[7]}$ and other large cohorts ${ }^{[8]}$ have underscored the public health problem of high CHD risk and increased prevalence of prediabetic states, such as different features of metabolic syndrome, in patients with schizophrenia. In late 2005, the International Diabetes Federation modified the metabolic syndrome criteria previously elaborated by the National Cholesterol Education Program, with an emphasis on abdominal adiposity as a necessary condition to be met, in addition to a minimum of two of the other four criteria. ${ }^{\left[{ }^{[9}\right.}$ While

Address for correspondence:

Dr. Jayanta Kumar Rout, Department of Biochemistry, R. G. Kar Medical College, Kolkata-700 004, W.B., India.

E-mail: dr.jayantarout@yahoo.in 
this newer definition asserts the primacy of central obesity in the conceptualization of metabolic syndrome, it also fuelled an ongoing debate in the recent literature over the value of the metabolic syndrome concept. ${ }^{[10]}$

Thus, it seems that there are considerable arguments whether obesity or WHR is affected by olanzapine, and that different ethnic groups may have different reactions. However, obesity has been an important contributing factor for developing medical problems like type 2 diabetes mellitus and metabolic syndrome, which constitute important risk factors for cardiovascular deaths in the schizophrenia patients. ${ }^{[11]}$ Goff et al,have suggested that clinicians treating schizophrenia patients with antipsychotics should properly monitor the cardiovascular risk factors, particularly for combating the metabolic syndrome, general obesity and abdominal obesity. ${ }^{[8]}$ Weight gain is worse with clozapine and olanzapine; minimal with aripiprazole and ziprasidone; and intermediate with other antipsychotics, including low-potency first generation antipsychotics (FGAs). ${ }^{[12]}$ Among the second generation antipsychotic drugs (SGA), olanzapine and clozapine are associated with excessive body weight gain, hyperglycemia, dyslipidemia and probably a pro-coagulant state. ${ }^{[13,14]}$ Olanzapine, although associated with a lesser amount of extra-pyramidal side effects, was found to cause a significant weight gain in bipolar mood disorder patients also. ${ }^{[15]}$ Obesity, particularly abdominal fat deposition indicated by a high waist hip ratio, has been a strong predictor of cardiovascular sequel such as coronary heart disease, myocardial infarction, or stroke. ${ }^{[16,17]}$ Although, several earlier studies as well as recent ones have indicated an overall increase in body fat, as indicated by an increased BMI, in schizophrenia patients stabilized on olanzapine or clozapine, ${ }^{[18-20]}$ some of them did not find any change in the waist hip ratio suggesting that the type of fat distribution was not affected by either of these drugs. ${ }^{[20]}$ On the other hand, another large study undertaken in India reported a significantly higher increase in the waist circumference and serum triglyceride in olanzapine treated patients. ${ }^{[21]} \mathrm{Kim}$ et al,reported that the relationship between BMI and insulin resistance was similar between the SGA treated schizophrenia and the reference population. ${ }^{[22]}$ Different results from different areas thus indicated that the physical indices vary from region to region based on their geographical distribution. Asian Indians have been a unique ethnic group in terms of body morphology and cardiovascular disease risk. In a recent large cross sectional study involving 933 European, Maori, Pacific Island and Asian Indian adult volunteers, Asian Indians were found to have more fat; both total and in the abdominal region, with less lean mass, skeletal muscle and bone mineral than all other ethnic groups. Furthermore, their abdominal fat was found to increase with advancing age with little changes in overall body fat. ${ }^{[23]}$ In a South Indian population group Kaur et al.found WHR to be the best predictor for type 2 DM. ${ }^{[24]}$ However, few studies are available regarding relative importance of the WHR and BMI as markers of dyslipidemia and insulin resistance in the schizophrenia patients stabilized on SGAs in the Indian population.

Keeping these factors in mind we hypothesized that dyslipidemia and insulin resistance could be better reflected and predicted by either overall body fat or abdominal adiposity in our region and hence, undertook the present study to evaluate it by assessing corresponding lipid parameters, physical indices of obesity and insulin resistance by homeostasis model assessment (HOMA) in such subjects.

\section{Materials and Methods}

\section{Study design}

The present study was designed as a hospital based case control study within the study period of November 2009 to December 2010. It was undertaken in the outpatient department (OPD) of Psychiatry and the department of Biochemistry of a government medical college and hospital in a rural district in West Bengal, India.

\section{Selection of cases and controls}

Cases were selected as sample of convenience from consecutive patients of schizophrenia of both sexes, receiving oral olanzapine treatment for at least six months from the Psychiatry outpatient department (OPD). Diagnosis of schizophrenia was done by Diagnostic and Statistical Manual of Mental Disorders (DSM) IV criteria. ${ }^{[25]}$ Patients were included provided they had no other DSM IV disorder that required antipsychotic treatment, had attained the age of 18 years and could give informed consent after understanding the objectives of the study. All selected cases were stable on an average dosage of 5-15 mg per day of olanzapine. Patients receiving benzodiazepines in requisite dosage in addition to olanzapine were also considered. Exclusion criteria were: 1) type 2 diabetes mellitus or a history of the disease among first degree relatives, 2) any somatic illness at the time of inclusion, neoplasm, metabolic or endocrine disorder or neurological diseases, 3) substantial use of alcohol or nicotine in any form within last 6 months or DSM IV criteria for alcohol dependence disorder, psychoactive substance abuse, or dependence disorder, 5) use of any antipsychotic medication other than olanzapine, and any other medication associated with insulin resistance (e.g. 
glucocorticoids, hydrochlorothiazides, furosemides, beta blockers, phenytoin, nicotinic acid etc.). All these criteria were included in a semi structured interview schedule.

Control subjects were selected from persons accompanying the patients in OPD who were free from any metabolic or psychiatric disorders. Relatives of the patients were excluded during this selection. Overall exclusion criteria were same as those used for case selection procedure. Additional exclusion criteria for the control group were any lifetime diagnosis of schizophrenia and/or major depressive illness, or any current Axis-I disorder. All participants were from a large rural base of Eastern India having approximately similar ethnicity, socioeconomic status and dietary habits. Written consents were obtained from all participants or their legal guardians. 38 patients (14 females) fulfilling the above inclusion and exclusion criteria were finally selected as cases for the study. Similarly, 30 age and sex (12 females) matched healthy people were recruited in the control group. The study followed the guideline of Helsinki declaration 1975 and was approved by the institutional ethical committee of the concerned Medical College and Hospital.

\section{Estimation of test parameters}

All parameters were assayed from $12 \mathrm{hr}$ fasting blood samples obtained from cases and controls. Fasting blood glucose (FBG), serum total cholesterol and serum triglyceride were assayed by glucose oxidase peroxidase method (GOD-POD), cholesterol oxidase phenylperoxidaseaminophenozonphenol (CHOD-PAP), and glycerol phosphate oxidase peroxidase (GPOPOD) method respectively. High density lipoprotein (HDL) cholesterol was measured by phosphotungstic acid precipitation method. We calculated low density lipoprotein (LDL) cholesterol according to the method of Friedewald and colleagues. The biochemical assays were done using the autoanalyzer ERBA XL 600. Internal quality control was performed for all parameters simultaneously. All test reagents were purchased from Ranbaxy RFCL, India and the quality control materials (Lyphochek, level 1 and 2) were purchased from Bio-Rad laboratories, USA.

Serum insulin was assayed by ELISA kit AccuBind from Monobind Inc. USA. This method has been reported to show a high degree of correlation (correlation coefficient 0.975) with reference radioimmunoassay method. No cross reactivity with C-peptide was detected. The serum was separated at $4^{\circ} \mathrm{C}$ and was stored at $-20^{\circ} \mathrm{C}$ till assay was done. Assays were done within one week from the date of collection in fully automated ELISA reader and washer from TECAN, Austria.
As a widely validated clinical and epidemiological tool for estimating insulin resistance and $\beta$ cell function, the homeostasis model assessment (HOMA) is derived from a mathematical assessment of the balance between hepatic glucose output and insulin secretion from fasting levels of glucose and insulin. ${ }^{[26,27]}$ This model requires only a single measurement of insulin and glucose in the basal state and so, in some conditions, is a suitable alternative for large-scale epidemiologic studies to the sophisticated "gold standard" methods which usually require dynamic data via costly and invasive procedures. Previous cross-sectional studies have shown that high HOMA-IR was associated with increased prevalence of impaired glucose tolerance (IGT) and type 2 diabetes in several population. ${ }^{[28]}$

The HOMA-IR in our present study was calculated with the help of HOMA2 Calculator v2.2 obtained from the website http://www.dtu.ox.ac.uk/homa. Body mass index (BMI) was calculated from the formula of weight $(\mathrm{kg}) /$ height $\left(\mathrm{m}^{2}\right)$ for both cases and controls.

\section{Statistical analysis}

The two matched groups were compared for differences between means of different test parameters by unpaired $\mathrm{t}$ tests [Table 1]. Analysis of co-variance (ANCOVA) was performed [Table 2] to find out the dependence of insulin resistance on FBG, BMI, WHR and lipid parameters in the case group after controlling the effects of age and sex. Significance was considered as $P<0.05$ for all statistical tests. All statistical analyses were done using SPSS software version 16.0 for Windows.

\section{Results}

Mean (SD) age in years for the cases and controls were 31.76 (7.82) and 35.46 (9.02) respectively, the difference being statistically insignificant $(\mathrm{t}=-1.78, P=0.08$, data not shown in Table). The male: Female ratios were 24:14 and 18:12 respectively in cases and controls. The distribution of males and females in both groups was found insignificant by Chi square test, the $\chi 2$ for males and females between both groups being $1.19(P=0.274)$ and $0.154(P=0.695)$ respectively (data not shown in Table).

The mean values for fasting serum insulin and insulin resistance indicator HOMA-IR showed a significant higher value $(P<0.001)$ in the case group without revealing any such change in FBG $(P=0.201)$ [Table 1]. Serum triglyceride level and LDL cholesterol levels were higher $(P<0.001$ and $P=0.034$ respectively) while the HDL cholesterol level was significantly lowered $(P=0.016)$ in the olanzapine treated group. Furthermore, WHR an 
Table 1: Distribution of descriptive measures in olanzapine treated case $(\mathrm{N}=38)$ and control $(\mathrm{N}=30)$ groups

\begin{tabular}{|c|c|c|c|c|}
\hline & $\begin{array}{l}\text { Cases mean } \\
\text { (SD) }\end{array}$ & $\begin{array}{l}\text { Controls } \\
\text { mean (SD) }\end{array}$ & t value & $P$ value \\
\hline $\begin{array}{l}\text { Fasting blood } \\
\text { glucose (mg/dl) }\end{array}$ & 101(18.03) & $96.2(13)$ & 1.2 & 0.201 \\
\hline Insulin (ulU/ml) & $18.23(5.78)$ & $10.26(3.96)$ & 6.3 & $<0.001^{*}$ \\
\hline HOMA-IR & $4.56((1.81)$ & $2.39(1.00)$ & 5.5 & $<0.001^{*}$ \\
\hline $\begin{array}{l}\text { Serum cholesterol } \\
(\mathrm{mg} / \mathrm{dl})\end{array}$ & $169.47(24.51)$ & $154.31(37.93)$ & 1.98 & 0.052 \\
\hline $\begin{array}{l}\text { Serum triglyceride } \\
(\mathrm{mg} / \mathrm{dl})\end{array}$ & $168.89(41.61)$ & $133.06(44.92)$ & 3.37 & $0.001^{*}$ \\
\hline $\begin{array}{l}\text { Serum LDLc } \\
(\mathrm{mg} / \mathrm{dl})\end{array}$ & $101.87(22.4)$ & 87.37(32.19) & 2.17 & $0.034^{*}$ \\
\hline $\begin{array}{l}\text { Serum HDLc } \\
(\mathrm{mg} / \mathrm{dl})\end{array}$ & $35.21(6.09)$ & $39.37(7.75)$ & -2.4 & $0.016^{*}$ \\
\hline BMI & $21.68(5.54)$ & 20.92(3.12) & 0.61 & 0.54 \\
\hline WHR & $0.92(0.06)$ & $0.88(0.05)$ & 2.4 & $0.017^{*}$ \\
\hline
\end{tabular}

indicator of abdominal obesity, showed a significant higher value $(P=0.017)$ in the case population whereas, no such change was found in the BMI $(P=0.54)$, an indicator of general obesity.

Analysis of co-variance revealed significant dependence of HOMA-IR on serum triglyceride ( $\mathrm{F}=0.016, P=0.016)$ and WHR ( $F=5.7, P=0.037$ ) [Table 2] only that indicated WHR to be a better anthropometric predictor than BMI for development of insulin resistance in the olanzapine treated case population.

\section{Discussion}

In our present study we found that schizophrenia cases stabilized on olanzapine therapy exhibited higher values for insulin resistance (HOMA-IR), serum triglyceride and LDL cholesterol along with a lower HDL cholesterol level, than did the matched control group. ANCOVA study revealed that the HOMA-IR in cases was dependent on the serum triglyceride level and WHR, but not on the BMI.

The impact of olanzapine on metabolic outcomes has been well documented, ${ }^{[29]}$ and the association between insulin resistance, serum triglycerides and central adiposity were found to be particularly strong for many years ${ }^{[30,31]}$ Although, some studies have attributed more importance to the overall body fat deposition (as reflected by an increased BMI) than the type of fat distribution (as reflected by an increased WHR) in olanzapine treated patients, ${ }^{[20]}$ abdominal fat deposition has been supposed to be a strong predictor of cardiovascular sequel such as coronary heart disease, myocardial infarction, or
Table 2: ANCOVA analysis controlling the effect of age and sex to show the dependence of the Insulin resistance (HOMA-IR) on various metabolic and anthropometric parameters in the olanzapine treated schizophrenia patients $(\mathrm{N}=38)$

\begin{tabular}{lcc}
\hline Tests of between-subjects effects & & \\
\hline Dependent variable: Homa-IR & & \\
Source & $\mathrm{F}$ & Sig. \\
Corrected model & 1.694 & 0.192 \\
Intercept & 5.888 & $0.036^{*}$ \\
Serum cholesterol (mg/dl) & 0.014 & 0.907 \\
Serum triglyceride $(\mathrm{mg} / \mathrm{dl})$ & 8.309 & $0.016^{*}$ \\
Serum LDLc $(\mathrm{mg} / \mathrm{dl})$ & 0.001 & 0.982 \\
Serum HDLc $(\mathrm{mg} / \mathrm{dl})$ & 0.541 & 0.479 \\
BMI & 0.000 & 0.997 \\
WHR & 5.770 & $0.037^{*}$ \\
Age (Years) & 0.665 & 0.775 \\
Sex & 0.108 & 0.749 \\
\hline R Squared=0.821 (Adjusted R Squared $=0.336)$ * $P<0.05$ &
\end{tabular}

stroke ${ }^{[16,17]}$ as well as the metabolic and blood pressure variables. ${ }^{[32]}$

In our study, abnormalities in the lipid metabolism in the case group was reflected by a significantly increased WHR, which is well in track with some recent large studies undertaken in the United States ${ }^{[7]}$ and India. ${ }^{[21]}$ In a Korean study also, Jeong et al,reported WHR to be more consistently related to the dyslipidemic changes than BMI. ${ }^{[33]}$ Furthermore, a significant dependence of the HOMA-IR on the serum triglyceride level and WHR, as observed in our study [Table 2], indicated a causal relationship between SGA induced dyslipidemia and insulin resistance. It has been suggested that an insulin resistant state accompanies the developmental process of schizophrenia ${ }^{[34-36]}$ that is further complicated by SGA induced dyslipidemia and other biochemical abnormalities. Kluge et al,has described an association of increased BMI in olanzapine treated patients with raised levels of serum leptin and cytokines. ${ }^{[20]}$ Reduced levels of adiponectin were suggested to play a role in inducing insulin resistance in olanzapine treated patients. ${ }^{[37]}$ Some authors have reported relationships between serotonin receptors HTR 2C and HTR 2A haplotypes with increased BMI and $\mathrm{C}$ peptide levels in these patients. ${ }^{[38]}$ Thus, although it is evident that within 3 months of exposure to olanzapine there is a significant increase in the proportion of subjects meeting the criteria of metabolic syndrome, ${ }^{[7]}$ the exact mechanism of this drug induced abnormal glucose metabolism remains unclear. ${ }^{[39-41]}$ This requires special mention for a need of monitoring the patients on long term olanzapine treatment to minimize the metabolic and cardiovascular consequences. Increased WHR and BMI have been strong predictors of increased body 
fat and cardiovascular sequel. ${ }^{[17,20]}$ Asian Indians are a unique ethnic group in terms of body morphology as for any given BMI they have higher central obesity and abdominal fat. Raji et al,reported that despite a similar BMI, an altered body composition with greater abdominal fat was associated with hyperinsulinemia, insulin resistance, and dyslipidemia in the Asian Indian population in comparison to their matched Caucasian counterparts. ${ }^{[42]}$ In a South Indian population group, Kaur et al,found WHR to be the best predictor for type $2 \mathrm{DM}^{[24]}$ In our study, the analysis of co-variance revealed that WHR predicted the HOMA-IR values more significantly $(\mathrm{F}=5.7, P=0.037)$ than did the BMI $(\mathrm{F}<0.001, P=0.0 .997)$ in the case group irrespective of their age and sex. Our findings, thus, extend these views by advocating that SGA induces a predominant abdominal fat deposition that, for any given BMI, turns out to be a major predictive factor for development of insulin resistance and dyslipidemia in schizophrenia patients stabilized on olanzapine in our region. An underlying purpose behind the identification of individuals who develop insulin resistance or manifest the physical indices of obesity, is to prevent the conversion from prediabetes to overt $\mathrm{DM}$, since type $2 \mathrm{DM}$ is considered a predictor of future myocardial infarction risk equivalent to having a diagnosis of CHD. ${ }^{[43]}$ However, early changes in the insulin resistance are not reflected by corresponding alterations in the FBG levels. It is not also practically feasible to measure insulin resistance periodically in all sections of patients in all setups. On the other hand, a periodic measurement of WHR is a much easier and more approachable protocol.

Moreover, our interpretation reiterates the dependence of these anthropometric markers on different cultural and ethnic backgrounds. Adult anthropometry has been recognized as a biomarker of 'early life' ${ }^{\text {[33] }}$ and several factors including genetic polymorphism of PPAR $\gamma$ gene $^{[44]}$ and some candidate genes for obesity and metabolic-related traits ${ }^{[45]}$ may influence the variable interpretations of the parameters like BMI and WHR depending on ethnicity and cultural background in different regions. Likewise, polymorphism in genes implicated in circadian regulation or its related downstream pathways have been recently found to play important roles in the regulation of antropomorphic parameters, metabolic profile and insulin resistance in patients with schizophrenia during long-term treatment with SGA ${ }^{[46]}$ These genetic polymorphisms stress on the fact that the obesity and dyslipidemia induced by the second generation antipsychotics depend largely on the allelic variations of several genes in the population. Our findings, hence, necessitate relevant research works in our region accordingly.

\section{Limitations}

A limitation of our study is that we did not perform any formal evaluation of the diet of the participant which might have influenced the FBG and insulin levels. However, as all participants were from a similar geographical area having similar ethnicity and socioeconomic status they had almost similar types of dietary habits. Hence, the dietary factors did not seem to influence the findings in this study, since body weight (as reflected by the BMI) between the case and control group did not show a significant difference.

\section{References}

1. Babidge NC, Buhrich N, Butler T. Mortality among homeless people with schizophrenia in Sydney, Australia: A 10-year follow-up. Acta Psychiatr Scand 2001;103:105-10.

2. Hiroeh U, Appleby L, Mortensen PB, Dunn G. Death by homicide, suicide, and other unnatural causes in people with mental illness: A population-based study. Lancet 2001;358:2110-2.

3. Morgan MG, Scully PJ, Youssef HA, Kinsella A, Owens JM, Waddington JL. Prospective analysis of premature mortality in schizophrenia in relation to health service engagement: A 7.5-year study within an epidemiologically complete, homogeneous population in rural Ireland. Psychiatry Res 2003;117:127-35.

4. Cohn T, Prud'homme D, Streiner D, Kameh H, Remington G. Characterizing coronary heart disease risk in chronic schizophrenia: High prevalence of the metabolic syndrome. Can J Psychiatry 2004;49:753-60.

5. Holt RI. Diagnosis, epidemiology and pathogenesis of diabetes mellitus: An update for psychiatrists. Br J Psychiatry Suppl 2004, 47:S55-63.

6. Saha S, Chant D, McGrath J. A systematic review of mortality in schizophrenia: Is the differential mortality gap worsening over time? Arch Gen Psychiatry 2007;64:1123-31.

7. Meyer JM, Davis VG, Goff DC, McEvoy JP, Nasrallah HA, Davis SM, et al. Change in metabolic syndrome parameters with antipsychotic treatment in the CATIE Schizophrenia Trial: Prospective data from phase 1. Schizophr Res 2008;101:273-86.

8. Goff DC, Cather C, Evins AE, Henderson DC, Freudenreich O, Copeland PM, et al. Medical morbidity and mortality in schizophrenia: Guidelines for psychiatrists. J Clin Psychiatry 2005;66:183-94; quiz 147, 273-4.

9. Alberti KG, Zimmet P, Shaw J: Metabolic syndrome--a new world-wide definition. A Consensus Statement from the International Diabetes Federation. Diabet Med 2006, 23:469-80.

10. Reaven GM. The metabolic syndrome: Requiescat in pace. Clin Chem 2005;51:931-8.

11. Birkenaes AB,Søgaard AJ,Engh JA,Jonsdottir H,Ringen PA,Vaskinn A, et al. Sociodemographic characteristics and cardiovascular risk factors in patients with severe mental disorders compared with the general population. J Clin Psychiatry 2006;67:425-33.

12. Gardner DM, Baldessarini RJ, Waraich P. Modern antipsychotic drugs: A critical overview. CMAJ 2005;172:1703-11.

13. Hägg S,Spigset O. Antipsychotic-induced venous thromboembolism: A review of the evidence. CNS Drugs 2002;16:765-76.

14. Newcomer JW. Metabolic considerations in the use of antipsychotic medications: A review of recent evidence. J Clin Psychiatry 2007;68:20-7.

15. Derry S, Moore RA. Atypical antipsychotics in bipolar disorder: Systematic review of randomised trials. BMC Psychiatry 2007;7:40.

16. Megnien JL, Denarie N, Cocaul M, Simon A, Levenson J. Predictive value of waist-to-hip ratio on cardiovascular risk events. Int J Obes Relat Metab Disord 1999;23:90-7.

17. Seidell JC, Pérusse L, Després JP, Bouchard C. Waist and hip circumferences have independent and opposite effects on cardiovascular disease risk factors: The quebec family study. Am J Clin Nutr 2001;74: 315-21.

18. Allison DB, Fontaine KR, Heo M, Mentore JL, Cappelleri JC, Chandler LP, 
et al. The distribution of body mass index among individuals with and without schizophrenia. J Clin Psychiatry 1999;60:215-20.

19. Himmerich H, Schuld A, Pollmacher T. [Weight gain during treatment with antipsychotics: Clinical relevance, pathophysiology, and therapeutical strategies]. Psychiatr Prax 2004;31:S233-7.

20. Kluge M, Schuld A, Schacht A, Himmerich H, Dalal MA, Wehmeier PM, et al. Effects of clozapine and olanzapine on cytokine systems are closely linked to weight gain and drug-induced fever. Psychoneuroendocrinology 2009;34:118-28

21. Saddichha S, Manjunatha N, Ameen S, Akhtar S. Metabolic syndrome in first episode schizophrenia-a randomized double-blind controlled, shortterm prospective study. Schizophr Res 2008;101:266-72.

22. Kim SH, Nikolics L, Abbasi F, Lamendola C, Link J, Reaven GM, et al. Relationship between body mass index and insulin resistance in patients treated with second generation antipsychotic agents. J Psychiatr Res 2010;44:493-8.

23. Rush EC, Freitas I, Plank LD. Body size, body composition and fat distribution: Comparative analysis of European, Maori, Pacific Island and Asian Indian adults. Br J Nutr2009;102:632-41.

24. Kaur P, Radhakrishnan E, Sankarasubbaiyan S, Rao SR, KondalsamyChennakesavan S, Rao TV, et al. A comparison of anthropometric indices for predicting hypertension and type 2 diabetes in a male industrial population of Chennai, South India. Ethn Dis 2008;18:31-6.

25. Diagnostic and Statistical Manual of Mental Disorders DSM-IV-TR. American Psychiatric Association 2004.

26. Matthews DR, Hosker JP, Rudenski AS, Naylor BA, Treacher DF, Turner RC. Homeostasis model assessment: Insulin resistance and betacell function from fasting plasma glucose and insulin concentrations in man. Diabetologia 1985;28:412-9.

27. Wallace TM, Levy JC, Matthews DR. Use and abuse of HOMA modeling. Diabetes Care 2004;27:1487-95

28. Ramachandran A, Snehalatha C, Viswanathan V, Viswanathan M, Haffner SM. Risk of noninsulin dependent diabetes mellitus conferred by obesity and central adiposity in different ethnic groups: A comparative analysis between Asian Indians, Mexican Americans and Whites. Diabetes Res Clin Pract 1997;36:121-5.

29. Pallava A, Chadda RK, Sood M, Lakshmy R. Metabolic syndrome in schizophrenia: A comparative study of antipsychotic-free/naïve and antipsychotic-treated patients from India. Nord J Psychiatry 2012;66:215-21.

30. Mayer-Davis EJ, Levin S, Bergman RN, D'Agostino RB Jr, Karter AJ, Saad MF; Insulin Resistance Atherosclerosis Study (IRAS). Insulin secretion, obesity, and potential behavioral influences: results from the Insulin Resistance Atherosclerosis Study (IRAS). Diabetes Metab Res Rev 2001;17:137-45

31. McLaughlin T, Abbasi F, Cheal K, Chu J, Lamendola C, Reaven G. Use of metabolic markers to identify overweight individuals who are insulin resistant. Ann Intern Med 2003;139:802-9.

32. Ghosh A. Interrelationship of waist circumference and subcutaneous fat with metabolic and blood pressure measures among Asian Indian men. Anthropol Anz 2007;65:75-85.

33. Jeong SK, Seo MW, Kim YH, Kweon SS, Nam HS. Does waist indicate dyslipidemia better than BMI in Korean adult population? J Korean Med Sci 2005;20:7-12

34. Cohn TA, Remington G, Zipursky RB, Azad A, Connolly P, Wolever TM. Insulin resistance and adiponectin levels in drug-free patients with schizophrenia: A preliminary report. Can J Psychiatry 2006;51:382-6.
35. van Nimwegen LJ, Storosum JG, Blumer RM, Allick G, Venema HW, de Haan L, et al. Hepatic insulin resistance in antipsychotic naive schizophrenic patients: Stable isotope studies of glucose metabolism. J Clin Endocrinol Metab 2008;93:572-7.

36. Dasgupta A, Singh OP, Rout JK, Saha T, Mandal S. Insulin resistance and metabolic profile in antipsychotic naïve schizophrenia patients. Prog Neuropsychopharmacol Biol Psychiatry 2010;34:1202-7.

37. Richards AA, Hickman IJ, Wang AY, Jones AL, Newell F, Mowry BJ, et al. Olanzapine treatment is associated with reduced high molecular weight adiponectin in serum: A potential mechanism for olanzapine-induced insulin resistance in patients with schizophrenia. J Clin Psychopharmacol 2006;26:232-7.

38. Gunes A, Melkersson KI, Scordo MG, Dahl ML. Association between HTR2C and HTR2A polymorphisms and metabolic abnormalities in patients treated with olanzapine or clozapine. J Clin Psychopharmacol 2009;29:65-8

39. American Diabetes Association; American Psychiatric Association; American Association of Clinical Endocrinologists;North American Association for the Study of Obesity. Consensus development conference on antipsychotic drugs and obesity and diabetes. J Clin Psychiatry 2004;65:267-72.

40. Engl J, Laimer M, Niederwanger A, Kranebitter M, Starzinger M, Pedrini MT, et al. Olanzapine impairs glycogen synthesis and insulin signaling in L6 skeletal muscle cells. Mol Psychiatry 2005;10:1089-96.

41. Fatemi SH, Reutiman TJ, Folsom TD, Bell C, Nos L, Fried P, et al. Chronic olanzapine treatment causes differential expression of genes in frontal cortex of rats as revealed by DNA microarray technique. Neuropsychopharmacology 20061;31:1888-99.

42. Raji A, Seely EW, Arky RA, Simonson DC. Body fat distribution and insulin resistance in healthy Asian Indians and Caucasians. J Clin Endocrinol Metab 2001;86:5366-71.

43. Expert Panel on Detection, Evaluation, and Treatment of High Blood Cholesterol in Adults. Executive summary of the third report of the national cholesterol education program (NCEP) expert panel on detection, evaluation, and treatment of high blood cholesterol in adults (Adult Treatment Panel III). JAMA 2001;285:2486-97.

44. Herken H, Erdal M, Aydin N, Sengul C, Karadag F, Barlas O, et al. The association of olanzapine-induced weight gain with peroxisome proliferator-activated receptor-gamma2 Pro12Ala polymorphism in patients with schizophrenia. DNA Cell Biol 2009;28:515-9.

45. Kuo PH, Kao CF, Chen PY, Chen CH, Tsai YS, Lu ML, et al. Polymorphisms of INSIG2, MC4R, and LEP are associated with obesity-and metabolic-related traits in schizophrenic patients. J Clin Psychopharmacol 2011;31:705-11.

46. Moons T, Claes S, Martens GJ, Peuskens J, Van Loo KM, Van Schijndel JE, et al. Clock genes and body composition in patients with schizophrenia under treatment with antipsychotic drugs. SchizophrRes 2011;125:187-93.

How to cite this article: Rout JK, Dasgupta A, Singh OP, Banerjee $\mathrm{U}$, Das B. Anthropometric parameters as indicators of metabolic derangements in schizophrenia patients stabilized on olanzapine in an Indian rural population. J Neurosci Rural Pract 2012;3:277-82. Source of Support: All reagents were procured by the Burdwan Medical College and Hospital authority as per rules and regulations of the government for routine patient care services. No other funding source was involved. Conflict of Interest: None declared. 